\title{
Medical Image of the Week: Boerhaave Syndrome
}
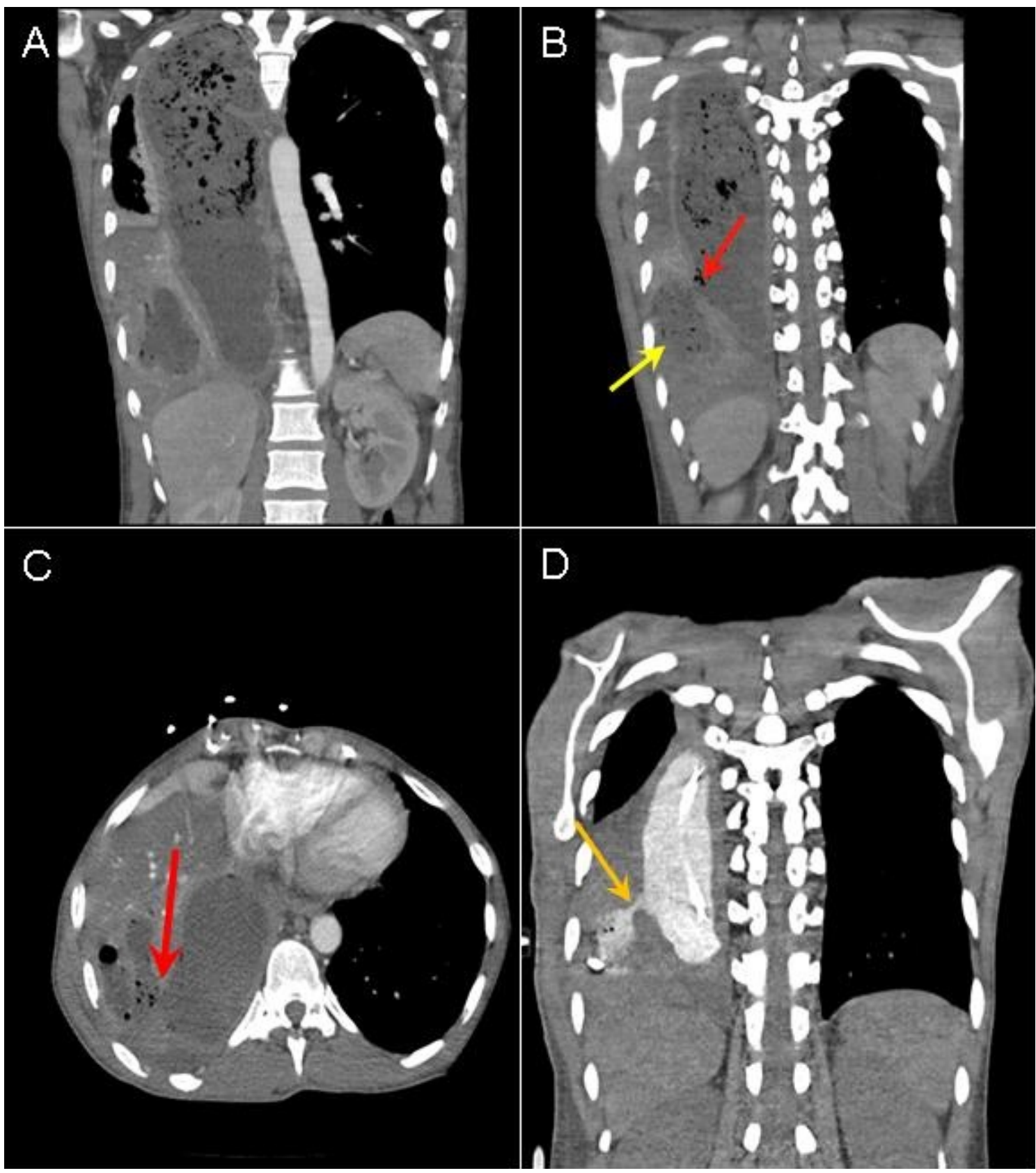

Figure 1. Panel A: Coronal CT image with IV contrast showing a massively dilated esophagus with retained food particles. Panel B: Coronal CT image depicting distal esophageal perforation (red arrow) rupturing into the lung parenchyma with resultant abscess formation (yellow arrow). Panel C: Axial image showing the dilated esophagus, ruptured into the lung (arrow). There is also mass effect on the mediastinum and heart.

Panel D. After insertion of a nasogastric tube and chest tube in the lung abscess, computed tomography was performed after administration of oral contrast. There is extravasation of contrast into the lung cavity which now contains a drainage catheter. Arrow shows the rupture site. 
A 41-year-old woman with a history of gastroesophageal reflux disease (GERD), asthma and iron deficiency anemia presented with complaints of right sided chest pain, nausea and emesis for several days prior to hospital presentation. She had also been experiencing progressive dysphagia to solids for a month preceding admission. CT chest imaging revealed mega-esophagus (Figure $1 \mathrm{~A}$ ) with rupture into the right lung parenchyma and resultant abscess formation (Figure 1B and $1 \mathrm{C}$ ). A subsequent echocardiogram also confirmed mitral valve endocarditis. An image-guided chest tube was placed in the abscess for drainage. Endoscopy was attempted but visualization was difficult due to the presence of retained food. Given her low albumin and poor nutritional state, a jejunostomy tube was placed. Follow up CT imaging with contrast through a nasogastric tube confirmed extravasation of esophageal contrast into the right lung parenchyma (Figure 1D).

Blood and sputum cultures grew Candida glabrata. She was initially started on broad spectrum antibiotics which were later tapered to Liposomal Amphotericin B and ampicillin-sulbactam. Following resolution of her fungemia and optimization of her nutritional status 2 months later, she underwent Ivor Lewis esophagectomy, pyloroplasty and serratus anterior muscle flap buttress to the remnant esophageal staple line. Pathology of the excised esophageal tissue revealed muscular hypertrophy and marked reduction of ganglion cells consistent with achalasia. There was also a segment of esophageal mucosal ulceration, acute inflammation and an area of perforation. Post-operative esophagram revealed no obstructions and contrast flowed without issue through the proximal esophagus into the gastroesophageal anastomosis and into the stomach. The patient did well and on discharge from the hospital was tolerating oral intake.

This case illustrates the multi-faceted approach sometimes required for successful treatment of Boerhaave syndrome, or rupture of the esophagus usually after emesis. Initial management included treating the patient's sepsis with appropriate antifungal therapy in addition to placing a jejunostomy tube for nutrition-a conservative approach which has proven successful in other reported cases (1). Following resolution of the fungemia, she underwent surgical repair for permanent treatment of her esophageal disease.

While the patient had underlying achalasia predisposing her to spontaneous esophageal rupture, Candida glabrata has also been reported to compromise the esophageal lining through angio-invasive mechanisms (2). Given the pathology findings of mucosal ulceration and inflammation of excised esophageal tissue, it is likely that the patient's Boerhaave syndrome was due to both a combination of achalasia and Candida glabrata esophageal infection.

Nour Parsa $\mathrm{MD}^{1}$, Bhupesh Pokhrel $\mathrm{MD}^{2}$, Arash Meshksar MD${ }^{3}$, Mark Meyer $\mathrm{MD}^{4}$, and Samuel Kim MD ${ }^{4}$

Departments of ${ }^{1}$ Medicine, ${ }^{2}$ Gastroenterology, ${ }^{3}$ Radiology, and ${ }^{4}$ Cardiothoracic Surgery, University of Arizona

Tucson, AZ USA 


\section{References}

1. Shen G, Chai Y, Zhang GF. Successful surgical strategy in a late case of Boerhaave's syndrome. World J Gastroenterol. 2014 Sep 21;20(35):12696-700. [CrossRef] [PubMed]

2. Tran HA, Vincent JM, Slavin MA, Grigg A. Esophageal perforation secondary to angio-invasive Candida glabrata following hemopoietic stem cell transplantation. Clin Microbiol Infect. 2003 Dec;9(12):1215-8. [CrossRef] [PubMed] 